\title{
Eine untere Grenze der Verweildauer ist erreicht!
}

\author{
The Lower Bound of Length of Stay has been Reached!
}

Bibliografie

Dol 10.1055/s-2007-986348

Psychiat Prax 2008; 35 :

269-270

(c) Georg Thieme Verlag KG

Stuttgart · New York .

ISSN 0303-4259

Korrespondenzadressen

Priv.-Doz. Dr. med.

Hermann SpießI

Bezirksklinikum Regensburg

Universitätsstraße 84

93053 Regensburg

hermann.spiessl@medbo.de
Die Rahmenbedingungen für die stationäre und teilstationäre Versorgung in psychiatrisch-psychotherapeutischen Kliniken haben sich in den letzten Jahren dramatisch verändert. Es besteht eine zunehmende Leistungsverdichtung, mehr Patienten müssen in kürzerer Zeit behandelt werden [1]. In der Kostendiskussion im stationären Bereich spielt die Verweildauer eine zentrale Rolle, da zumindest bei vordergründiger Betrachtungsweise Einzelfallverweildauer und Fallkosten eng miteinander korrelieren und über diesen Indikator direkt auf die von den Kostenträgern zu erstattenden Kosten der Behandlung eingewirkt werden kann.

Dabei ist die Verweildauer als Kriterium der Effizienz einer Behandlung sehr umstritten. Krankenhausvergleiche hinsichtlich der Verweildauer sind mit methodologischen Problemen behaftet und erfordern eine angemessene Berücksichtigung relevanter Einflussvariablen [2,3]. Trotz aller Variabilität findet sich national und international ein Rückgang der Verweildauer [4], in psychiatrisch-psychotherapeutischen Kliniken in Deutschland von 66,5 Tagen (1991) auf 24,7 Tage (2004); dies entspricht einer prozentualen Veränderung von $-63 \%$ [1]!

Die zentrale Frage ist: Ist die Verkürzung der Verweildauer auf eine verbesserte Behandlungseffizienz zurückzuführen, oder werden die Patienten früher entlassen, obwohl sie eines längeren Aufenthaltes in der Klinik bedurft hätten? Letzteres könnte darauf beruhen, dass die Patienten jetzt problemlos in eine in der Versorgungsregion bestehende qualifizierte ambulante Nachsorge entlassen werden können. Eine andere Erklärung könnte aber auch der zunehmende Druck der Kostenträger auf die Kliniken sein, gekennzeichnet durch vermehrte Anforderung von Verlängerungsanzeigen (nach §301 SGB V), MDK-Fehlbelegungsprüfungen und Prüfungen durch sog. Einigungsausschüsse.
Die im Rahmen der Versorgungsforschung erhobenen Daten liefern einige Argumente bezüglich der Beantwortung dieser Frage: Während auf ältere Studien bezogene Metaanalysen keinen Effekt der Verweildauer auf die Wiederaufnahmerate oder auf andere Outcome-Maße fanden $[5,6]$, belegen alle neueren Studien - mit Ausnahme einer Studie bei Patienten mit affektiven Störungen [7] - einen Zusammenhang zwischen kürzerer Verweildauer und höherer Wiederaufnahmerate [8-13].

Um die Zusammenhänge zwischen Verweildauer, Wiederaufnahmerate und Behandlungserfolg genauer zu analysieren, wurden in einer eigenen Studie die Daten der psychiatrischen Basisdokumentation (BADO) von 18074 Patienten ausgewertet, die von 1995 bis 2003 vollstationär in der Psychiatrischen Klinik am Bezirksklinikum Regensburg behandelt wurden [14]. Als wesentliche Ergebnisse fanden sich: Im Verlauf der Jahre liegt bei Aufnahme ein zunehmend geringeres psychosoziales Funktionsniveau (GAF bei Aufnahme) vor. Trotz einer immer kürzeren Verweildauer konnte eine relativ größere Besserung (GAF bei Entlassung minus GAF bei Aufnahme) durch die stationäre Behandlung erzielt werden. Aufgrund des kränkeren Zustands bei Aufnahme wurden die Patienten dennoch in einem kränkeren Zustand (GAF bei Entlassung) entlassen. Mit der im Verlauf der Jahre kürzer werdenden Verweildauer stieg gleichzeitig die Wiederaufnahmerate an. Dieser Anstieg war besonders stark ausgeprägt bei Indexaufenthalten von drei bis zehn Tagen; er findet sich besonders bei Suchterkrankungen (ICD-10: F1) sowie bei Schizophrenien/wahnhaften Störungen (ICD-10: F2). Über alle Jahre hinweg zeigte sich, dass bei Fällen mit einer längeren Verweildauer eine größere Besserung (CGI Teil 2) erzielt wird, wobei sich bei Schizophrenien/wahnhaften Störungen (ICD-10: F2) sowie bei affektiven Störungen (ICD-10: F3) bei sechs Wochen ein Plateau fand. Bei Suchterkran- 
kungen (ICD-10: F1) war bei längerer Verweildauer (über zehn Tage) eine vermehrte Besserung festzustellen. Die mittlere kumulierte Verweildauer (innerhalb von 365 Tagen) blieb in den Jahren 1999 bis 2003 gleich.

Die auf Basis der psychiatrischen Basisdokumentation gewonnenen Daten belegen den Befund, dass eine (unter dem Kostendruck) zunehmend kürzere Verweildauer zu einer erhöhten Wiederaufnahmerate führt und ab einem gewissen Punkt die kumulierte Verweildauer (innerhalb eines Jahres) unverändert bleibt, was sich auch in vielen anderen Studien - insbesondere bei schizophrenen und suchtkranken Patienten - zeigte $[2,11,12,15]$. Die intendierte Kostenersparnis aufseiten der Krankenkassen wird somit nicht erreicht, der „Drehtüreffekt“ nimmt aber zu. Diese Veränderungen erfolgen auf Kosten der Patienten, die bei immer kürzer werdender Verweildauer pro Aufenthalt in zunehmend kränkerem Zustand entlassen werden. Dass die psychosoziale Leistungsfähigkeit bei Entlassung nur relativ gering abgefallen ist, ist dadurch bedingt, dass während des Aufenthaltes eine relativ größere Besserung erzielt werden konnte, d.h. dass die stationär-psychiatrische Behandlung in den letzten Jahren effektiver und effizienter geworden ist. Die effizientere Behandlung konnte bei zu kurzer Verweildauer den Abfall der psychosozialen Leistungsfähigkeit bei Entlassung jedoch - zumindest bislang - nicht vollständig kompensieren. Ein niedriges psychosoziales Funktionsniveau, ein instabiles Zustandsbild oder eine schwerere Krankheitssymptomatik bei Entlassung sind aber relevante Faktoren für eine häufigere Rehospitalisation [16-18]. Und hier schließt sich der Kreis: kürzere Verweildauer, kränkerer Entlassungszustand, häufigere Wiederaufnahme.

Als Schlussfolgerung ergibt sich, dass die Verkürzung der Verweildauer pro Aufenthalt eine untere Grenze erreicht hat und auf Kosten der Wiederaufnahmerate (also eines Krankheitsrückfalls) und anderer Outcome-Maße (also der Gesundheit des Patienten) geht. Ein weiterer Druck der Kostenträger auf die Verweildauer pro Einzelaufenthalt erscheint nicht sinnvoll, da das in den letzten Jahren gleichbleibende Niveau der mittleren kumulierten Verweildauer (und damit der Summe der tatsächlichen stationären Kosten) Indiz dafür ist, dass die Grenze für eine weitere Senkung der Einzelfallverweildauer erreicht ist. Sinnvoller erscheint dagegen, mit den regionalen Vertretern der Krankenkassen einen „runden Tisch“ zu bilden, datenbasiert die Versorgung in der Region zu analysieren und angemessene Überprüfungszeiträume der stationären Behandlungsnotwendigkeit zu vereinbaren. Dies würde nicht nur den Patienten zugute kommen, sondern auch den hohen administrativen Aufwand beider Seiten verringern.

\section{Literatur}

1 Schmidt-Zadel R, Kunze H. Brief an die Bundesministerin für Gesundheit. Nervenarzt 2006; 77: 1537 - 1539

2 Frick U, Rehm J, Krischker S, Cording C. Length of stay in a German psychiatric hospital as a function of patient and organizational characteristics - a multilevel analysis. Int J Meth Psychiatr Res 1999; 8: 146 191

3 Sitta P, Brand S, Schneider F, Gaebel W, Berger M, Farin E, Härter M. Faires Benchmarking der Behandlungsdauer depressiver Patienten in psychiatrisch-psychotherapeutischen Kliniken. Psychother Psych Med 2006; 56: $128-137$

4 Richter D. Die Dauer der stationären psychiatrischen Behandlung. Eine Übersicht über Methodik, Einflussfaktoren und Auswirkungen. Fortschr Neurol Psychiat 2001; 69: 19-31

5 Johnstone P, Zolese G. Systematic review of the effectiveness of planned short hospital stays for mental health care. Br Med J 1999; 318: $1387-1390$

6 Pfeiffer S, O'Malley DS, Shott S. Factors associated with the outcome of adults treated in psychiatric hospitals: A synthesis of findings. Psychiatr Serv 1996; 47: $263-269$

7 Lyons J, O'Mahoney MT, Miller SI, Neme J, Kabat J, Miller F. Predicting readmission to the psychiatric hospital in a managed care environment: Implications for quality indicators. Am J Psychiatry 1997; 154: $337-$ 340

8 Appleby L, Luchins DJ, Desai PN, Gibbons RD, Janicak RG, Marks R. Length of stay and recidivism among patients with schizophrenia. Psychiatr Serv 1996; 47: 985 - 990

9 Dickey B, Normand SLT, Norton EC, Azeni H, Fisher W, Altaffer F. Managing the care of schizophrenia: Lessons from a 4-year Massachusetts Medicaid study. Arch Gen Psychiatry 1996; 53: 945 - 952

10 Heeren O, Dixon L, Gavirneni S, Regenold WT. The association between decreasing length of stay and readmission rate on a psychogeriatric unit. Psychiatr Serv 2002; 53: 76-79

11 Menke T, Ashton CM, Petersen NJ, Wolinsky FD. Impact of an all-inclusive Diagnosis Related Group payment system on inpatient utilization. Med Care 1998; 36: 1126-1137

12 Richter D, Eikelmann B. Verweildauerrückgang bei stationären Behandlungen in der Psychiatrie: Positive oder negative Konsequenzen? Spektrum 2000; 29: 67-72

13 Rosenheck R, Massari L. Psychiatric inpatient care in the VA: Before, during and after DRG-based budgeting. Am J Psychiatry 1991; 148: $888-891$

14 Spießl H, Binder H, Cording C, Klein HE, Hajak G. Klinikpsychiatrie unter ökonomischem Druck. Dt Ärztebl 2006; 103: 2549- 2552

15 Goldstein G, Shemansky WJ. Length and number of hospitalization on two cohorts of veterans with chronic schizophrenia. Psychiatr Serv 2000; $51: 245-247$

16 Mojtabai B, Nicholson RA, Neesmith DH. Factors affecting relapse in patients discharged from a public hospital: results from survival analysis. Psychiatr Q 1997; 68: 117-129

17 Monnelly EP. Instability before discharge and previous psychiatric admissions as predictors of early readmission. Psychiatr Serv 1997; 48: $1584-1586$

18 Sweet $C$. Symptom severity and number of previous psychiatric admissions as predictors of readmission. Psychiatr Serv 1995; 46: 482 485 